\title{
Conditional moment closure for chemical reactions in laminar chaotic flows
}

\author{
A. Vikhansky* $\quad$ S. M. Cox ${ }^{\dagger}$
}

May 8, 2007

\begin{abstract}
We implement conditional moment closure (CMC) for simulation of chemical reactions in laminar chaotic flows. The $\mathrm{CMC}$ approach predicts the expected concentration of reactive species, conditional upon the concentration of a corresponding nonreactive scalar. Closure is obtained by neglecting the difference between the local concentration of the reactive scalar and its conditional average. We first use a Monte Carlo method to calculate the evolution of the moments of a conserved scalar; we then reconstruct the corresponding probability density function and dissipation rate. Finally, the concentrations of the reactive scalars are determined. The results are compared (and show excellent agreement) with full numerical simulations of the reaction processes in a chaotic laminar flow.
\end{abstract}

\section{Introduction}

Numerical simulation of mixing-sensitive chemical reactions in flows with high Péclet number is a nontrivial task. For decades, most research has concentrated on turbulent reactive flows, especially turbulent combustion. It is therefore no surprise that, at present, turbulent combustion models represent a powerful predictive tool. However, recent progress in microfluidic applications, for example, turns our attention away from turbulent processes and towards laminar reacting flows. Many microreactors are operated at low Reynolds numbers, but at high Péclet numbers ${ }^{1}$, and this combination of parameters poses particular difficulties for efficient chemical reaction from a segregated initial state, because the low Reynolds numbers make turbulence impossible, and the high Péclet numbers limit the efficiency of molecular diffusion. Fortunately, it is now well established that effective mixing can be achieved in the necessarily laminar flows that are realized at low Reynolds numbers, provided the Lagrangian particle paths are made chaotic $^{2,3,4}$.

\footnotetext{
*Department of Engineering, Queen Mary, University of London, Mile End Road, London E1 4NS, United Kingdom, E-mail: a.vikhansky@qmul.ac.uk.

†School of Mathematical Sciences, University of Nottingham, University Park, Nottingham NG7 2RD, United Kingdom, E-mail: stephen.cox@nottingham.ac.uk.
} 
Such chaotic flows rapidly generate, from an initially segregated distribution of reactants, an intertwined pattern of highly complicated morphology. In three dimensions, sheet-like "lamellar" structures are formed in some regions; reduced models for reaction in the flow are then commonly based on "straightening out" the lamellae into a one-dimensional array, leading to so-called lamellar models ${ }^{5,6,7}$. Although admittedly limited in scope, a recent evaluation of some simple lamellar models by one of us $^{8}$ has revealed shortcomings in the quantitative predictive power of such models. It is therefore appropriate to seek more accurate reduced models.

The obvious similarity between turbulent flows and laminar chaotic advection (which is also sometimes called "Lagrangian turbulence") suggests that one might reasonably borrow (with some modifications) ideas from turbulent combustion to simulate laminar chaotic reacting flows. In this spirit, we apply in this paper one of the most promising methods for modelling turbulent nonpremixed flames - namely, conditional moment closure $(\mathrm{CMC})^{9}$ — for laminar chaotic flows.

One might legitimately ask why such modelling is necessary for chemical reaction in a simple laminar flow, such as contemplated here. The reason is that, although the Eulerian velocity field is readily computed to high accuracy by traditional means such as finite elements or finite volumes (indeed, here we shall specify the flow field ab initio), the corresponding Lagrangian flow structures decrease in thickness exponentially in time. Hence, when the Péclet number is large, the Lagrangian flow structures lead to particular numerical difficulties.

Our focus is on the evolution of a multicomponent reaction in a laminar flow field $\boldsymbol{u}(t, \boldsymbol{x})$ in some region $\Sigma$. The combined effects of advection, diffusion and reaction are described by equations of the form

$$
\frac{\partial C_{i}}{\partial t}+\boldsymbol{u} \cdot \nabla C_{i}-D \nabla^{2} C_{i}=\sum_{j} \nu_{i j} \Upsilon_{j}
$$

where $C_{i}$ is the molar concentration of the $i$ th component, $D$ is the coefficient of diffusion (assumed equal for all species), $\nu_{i j}$ is a stoichiometric coefficient (with an appropriate sign) for the $i$ th component in the $j$ th reaction and $\Upsilon_{j}$ is the reaction rate (in $\mathrm{mol} / \mathrm{cm}^{3} \mathrm{~s}$ ) of the $j$ th reaction. We assume that the flow field $\boldsymbol{u}$ is unaffected by any changes in the local chemical composition or by the reaction process itself. Using (respectively) $L, U, C$ and $Y$ as characteristic scales for length, velocity, concentration, and reaction rate, we nondimensionalize the variables as follows: $\boldsymbol{u}^{\prime}=\boldsymbol{u} / U, \boldsymbol{x}^{\prime}=$ $\boldsymbol{x} / L, t^{\prime}=t U / L, \boldsymbol{C}^{\prime}=\boldsymbol{C} / C, \Upsilon_{j}^{\prime}=\Upsilon_{j} / Y$, where $\boldsymbol{C}=\left(C_{1}, C_{2}, \ldots\right)$ is the vector of concentrations. Then Eq. (1) becomes

$$
\frac{\partial C_{i}^{\prime}}{\partial t^{\prime}}+\boldsymbol{u}^{\prime} \cdot \nabla^{\prime} C_{i}^{\prime}-\frac{1}{\mathrm{Pe}} \nabla^{\prime 2} C_{i}^{\prime}=\frac{\mathrm{Da}}{\mathrm{Pe}} \sum_{j} \nu_{i j} \Upsilon_{j}^{\prime} \equiv \Omega_{i}\left(\boldsymbol{C}^{\prime}\right)
$$

Here the dimensionless parameters are the Damköhler and Péclet numbers, Da $=$ $Y L^{2} / D C$ and $\mathrm{Pe}=L U / D ; \Omega_{i}$ is dimensionless production/consumption rate of the $i$ th species. From this point, the problem is treated exclusively in its dimensionless 
form, and we remove the primes from all dimensionless variables, for the sake of simplicity.

The main hypothesis of CMC is that variations in the concentrations of the reactants in (2) are related to corresponding variations of one passive scalar ${ }^{9}$. Specifically, if the reactants are supplied to the system through two separate inlets, we define a variable $Z$ which is a normalized mixture fraction of a (nonreactive) tracer introduced through one of the inlets. The evolution of $Z$ is then governed by

$$
\frac{\partial Z}{\partial t}+\boldsymbol{u} \cdot \nabla Z-\frac{1}{\mathrm{Pe}} \nabla^{2} Z=0
$$

The expected value of the $i$ th (reactive) scalar conditioned on the mixture fraction is

$$
Q_{i}(\eta)=\left\langle C_{i} \mid Z=\eta\right\rangle
$$

where $\eta$ is sample space variable for the mixture fraction. This conditional mean evolves in $\eta$-space according to

$$
\frac{\partial Q_{i}}{\partial t}=N^{2}(\eta) \frac{\partial^{2} Q_{i}}{\partial \eta^{2}}+\Omega_{i}(\boldsymbol{Q}),
$$

where

$$
N^{2}(\eta)=\mathrm{Pe}^{-1}\left\langle(\nabla Z)^{2} \mid Z=\eta\right\rangle
$$

is the conditional scalar dissipation rate. The probability density function (PDF) $P$ for the mixture fraction satisfies

$$
\frac{\partial P(\eta)}{\partial t}=-\frac{\partial^{2} N^{2}(\eta) P(\eta)}{\partial \eta^{2}} .
$$

The mixture fraction is constrained to lie within a certain interval, and at the ends of this interval the PDF satisfies the boundary conditions

$$
N^{2}(\eta) P(\eta)=0, \frac{\partial N^{2}(\eta) P(\eta)}{\partial \eta}=0 .
$$

In the CMC derivation it is assumed that the conditional fluctuations $c_{i}=C_{i}-Q_{i}(\eta)$ are small and can be neglected. Note that in the limit case $\mathrm{Pe}=\infty$, each molecule is attached to its fluid element, and two particles which have identical chemical composition at the beginning of the process follow the same path in the composition space, i.e., $c_{i}=0$. Therefore, although $\mathrm{CMC}$ was initially developed for turbulent flows, the main assumption - which leads to Eqs. (5)-(8) - holds for any flows with high Pe.

The central problem in implementation of CMC is modelling the conditional scalar dissipation rate $N^{2}(\eta)$ and corresponding PDF $P(\eta)$. The most popular approach used in turbulent combustion is based on a presumed (usually two-parameter) PDF and a consistent model for $N^{2}(\eta)^{10,11,12}$. In the present work we use to our advantage the fact that the transport equation (3) in a laminar flow is simpler than the corresponding equation for a turbulent flow field. We use a Monte Carlo method to calculate the 
time evolution of moments of $Z$. Then we apply a special technique to reconstruct the $P(\eta)$ and $N^{2}(\eta) P(\eta)$. Once the mixing properties of the flow are known, Eq. (5) can be solved for an arbitrary chemical reaction. In order to characterize the errors of the method, we compare the approximate solution obtained using CMC with numerical simulations of the full advection-diffusion-reaction equations.

\section{Consistent modelling of PDF and scalar dissipation}

We use the similarity between the advection-diffusion equation (3) and the FokkerPlanck equation of a diffusive process ${ }^{13,14,15}$. Linear functionals of the solution of Eq. (3) can then be estimated by a statistical simulation using a Monte Carlo method, and the required computational resources do not depend on Pe. To develop the Monte Carlo method, we consider Eq. (3) as the forward Kolmogorov or Fokker-Planck equation of a stochastic process, for which the corresponding backwards Kolmogorov equation reads

$$
\frac{\partial Z}{\partial \tau}-\boldsymbol{u} \cdot \nabla Z-\frac{1}{\mathrm{Pe}} \nabla^{2} Z=0
$$

where $\tau=-t$ is backwards-directed time (and we have made use of the incompressibility of $\boldsymbol{u}$ in deriving (9)). In order to calculate the quality of the mixing at a point $\boldsymbol{x}^{0}$ at a time $t$, we track a tracer particle backwards in time according to the stochastic differential equation that corresponds to Eq. (9), i.e.,

$$
d \boldsymbol{x}(\tau)=-\boldsymbol{u}(\tau, \boldsymbol{x}) d \tau+\sqrt{\frac{2}{\mathrm{Pe}}} d \boldsymbol{\Xi}, \boldsymbol{x}(t)=\boldsymbol{x}^{0},
$$

where $d \boldsymbol{\Xi}$ is the increment of a Wiener process with unit dispersion. The concentration at the point $\boldsymbol{x}^{0}$ can be estimated by the Monte Carlo method as $Z\left(\boldsymbol{x}^{0}\right)=\langle\xi\rangle$, where

$$
\begin{cases}\xi=1, & \boldsymbol{x}(0) \in \Sigma_{1}, \\ \xi=-1, & \boldsymbol{x}(0) \in \Sigma_{2},\end{cases}
$$

and $\langle\cdots\rangle$ means mathematical expectation; $\Sigma_{1}$ and $\Sigma_{2}$ denote two subsets of the domain $\Sigma$, in which different reactants are initially placed.

Consider two statistically independent diffusion trajectories, each starting at the point $\boldsymbol{x}^{0}$ at a given time. Since they are independent, the corresponding values of $\xi$ for the two trajectories satisfy

$$
\left\langle\xi_{1} \xi_{2}\right\rangle=\left\langle\xi_{1}\right\rangle\left\langle\xi_{2}\right\rangle=Z^{2}\left(\boldsymbol{x}^{0}\right)
$$

Thus, only two random trajectories suffice to obtain an unbiased Monte Carlo estimate for the variation of the concentration at a point. In order to calculate the third moment we need three trajectories, etc.

Therefore, the Monte Carlo algorithm for the calculation of the $n$th moment $M_{n}=$ $\left\langle Z^{n}\right\rangle$ reads as follows: 
1. Generate a point $\boldsymbol{x}^{0}$ from a uniform distribution.

2. Track a random trajectory (10) with the initial condition $\boldsymbol{x}^{0}$.

3. Repeat step $2 n$ times and get an unbiased estimate for $Z^{n}\left(\boldsymbol{x}^{0}\right)$.

4. Repeat steps $1-3 N$ times $(N \gg 1)$ and average the results obtained.

It is clear that we may use the same trajectories in the calculation of different moments.

Once the moments $M_{n}(t)$ of the mixture fraction are known, we can use this information to approximate the PDF and corresponding scalar dissipation rate. We rewrite Eq. (7) in the form

$$
\frac{\partial P(\eta)}{\partial t}=-\frac{\partial^{2} W(\eta)}{\partial \eta^{2}}
$$

where $W(\eta)=N^{2}(\eta) P(\eta)$ is a fractional dissipation rate, i.e., $W(\eta) \Delta \eta$ is the amount of dissipation that occurs in the interval $[\eta, \eta+\Delta \eta]$. From the numerical point of view, Eq. (12) has two advantages over Eq. (7) itself: (i) we do not need to solve an equation with negative coefficient of diffusion; (ii) the equation becomes linear with respect to both functions involved, a fact which we shall use below. The boundary conditions for Eq. (12) are, from (8),

$$
\left.W\right|_{\eta= \pm 1}=0,\left.\frac{\partial W}{\partial \eta}\right|_{\eta= \pm 1}=0 .
$$

Multiplication of Eq. (12) by $\eta^{n}$ and integration by parts, using the boundary conditions (13), yield the following equation for $n$th moment:

$$
\theta_{n} \equiv \frac{d M_{n}}{d t}+n(n-1) \int_{-1}^{1} W(\eta) \eta^{n-2} \mathrm{~d} \eta=0
$$

Note from this equation that, as we expect for a passive scalar, mixing does not affect the first moment, i.e., the mean. The obvious constraints which should be imposed on $W(\eta)$ and $P(\eta)$ are that

$$
P(\eta) \geqslant 0 \text { and } W(\eta) \geqslant 0 .
$$

Then we require that at each instant the pair $P(\eta), W(\eta)$ minimizes the functional

$$
\Theta=\sum_{n} \theta_{n}^{2}+\alpha_{1} \int_{-1}^{1}\left(\frac{\partial^{2} W}{\partial \eta^{2}}\right)^{2} \mathrm{~d} \eta+\alpha_{2} \int_{-1}^{1}\left(\frac{\partial^{2} P}{\partial \eta^{2}}\right)^{2} \mathrm{~d} \eta
$$

subject to the constraints (12), (13) and (15); here $\alpha_{i}$ are (small) regularization parameters, which are necessary to make the above functional positively defined and smooth the solution.

Using time and mixture fraction increments $\Delta t$ and $\Delta \eta$ we discretize Eq. (12) in time and space as follows:

$$
\frac{P_{j}^{i+1}-P_{j}^{i}}{\Delta t}=-\frac{W_{j+1}^{i+1 / 2}-2 W_{j}^{i+1 / 2}+W_{j-1}^{i+1 / 2}}{2 \Delta \eta},
$$


where the upper and lower indices correspond to time and mixture fraction, respectively. Eqs. (14) and (16) are discretized in the same way. Since the constraints are linear, the resulting discrete optimization problem can be easily solved by a standard method for quadratic programming, and we do not discuss the solution method in detail.

To check the proposed algorithm, we solved a test problem for which the PDF consists of two Dirac $\delta$-functions which converge linearly with time, i.e.,

$$
P(t, \eta)=\frac{1}{2} \delta\left(\eta-\left(-1+\frac{t}{10}\right)\right)+\frac{1}{2} \delta\left(\eta-\left(1-\frac{t}{10}\right)\right) .
$$

It follows from Eq. (12) that

$$
W(t, \eta)=\frac{1}{20} H\left(\eta-\left(-1+\frac{t}{10}\right)\right)+\frac{1}{20} H\left(\eta-\left(1-\frac{t}{10}\right)\right),
$$

where $H$ is the Heaviside step function. Fig. 1 shows that the proposed numerical algorithm performs well in reproducing these results. The numerical method does not require any special treatment of a $\delta$-function: due to the discrete nature of Eq. (17) it is smeared over a small region of order $\Delta \eta$.

Of course, in a real situation, the Monte Carlo method provides the moments of the passive scalar only at a (small) number of discrete points, and so some interpolation procedure is necessary to approximate the time evolution of $M_{n}(t)$. We shall discuss this issue in the next section.

\section{Results and discussion}

In the present study we consider chemical reactions in the (two-dimensional) "sine flow" 16 . The (dimensionless) fluid domain is a periodic square box $0 \leqslant x, y \leqslant 1$ (with sides $x=0,1$ identified, and sides $y=0,1$ likewise). The flow is time-periodic, with period $T$; then ${ }^{16}$

$$
\boldsymbol{u}= \begin{cases}(\sin 2 \pi y, 0) & m T \leqslant t<\left(m+\frac{1}{2}\right) T \\ (0, \sin 2 \pi x) & \left(m+\frac{1}{2}\right) T \leqslant t<(m+1) T\end{cases}
$$

for $m=0,1,2, \ldots$. The flow is illustrated in Fig. 2. The case $T=1.6$ gives a flow that is "globally chaotic", in the sense that any regular islands are barely discernible to the eye in a Poincaré section; for other values of $T$, significant islands coexist with chaotic regions of the flow. The segregated initial state for Eq. (3) is given by

$$
Z(0, x, y)=\left\{\begin{array}{cc}
1 & 0 \leqslant x<\frac{1}{2} \\
-1 & \frac{1}{2} \leqslant x<1
\end{array}\right.
$$

In Fig. 3 we show, from direct simulation of the governing equation (3), the evolution of $Z$ for the case $T=1.0$, for which there are significant nonchaotic islands in the 
flow, associated with some period-2 periodic points. Results are shown for two Péclet numbers: $\mathrm{Pe}=10^{5}$ and $10^{3}$ (the numerical method is discussed later).

Monte Carlo calculations of the even moments of the passive scalar $Z$ are shown in Fig. 4 (odd moments vanish due to the symmetry of the initial conditions). Note that the standard deviation of one Monte Carlo estimate (11) is unity, and in order to calculate a moment whose value is $10^{-2}$ with an error of about $1 \%$ we need to simulate about $10^{6}$ random trajectories. A desktop PC can accomplish these calculations within an hour. However, if the moment is about $10^{-4}$ then we need four orders of magnitude more CPU time. Fortunately, as the mixing proceeds, the distribution of the passive scalar approaches a normal distribution. Thus we adopt the following strategy. We calculate the second moment by the Monte Carlo method. Other moments are calculated until they drop below $10^{-2}$, then we assume that these moments obey the same law as those of a normal distribution, namely, $M_{n}=(n-1) M_{2} M_{n-2}$. We use cubic splines to interpolate the obtained data. Note that the flow-field is time periodic and the mixing is due to the repeated reorientation and stretching of the interface. Therefore the dissipation rate can be a non-monotonic function of time, while the proposed interpolation procedure neglects the variations of the dissipation rate during one period, which can lead to some error, especially at the beginning of the process.

Once the moments $M_{n}(t)$ are calculated, we apply our reconstruction procedure in order to calculate the PDF $P$ and the fractional dissipation rate $W$. The shapes of the calculated functions depend on the number of moments used in the calculations. The results are presented in Fig. 5. As mentioned above, for $T=1.6$ the flow field is "globally chaotic", therefore the mixing is fast and the PDF quickly approaches a unimodal shape. For $T=1.0$, by contrast, the well-mixed chaotic region coexists with less-well mixed regular islands (see Fig. 3). The local peaks at $\eta= \pm 1$, which correspond to these poorly mixed regions, persist for a long time when $\mathrm{Pe}=10^{5}$, while the central peak (due to the chaotic region) shrinks in width exponentially with time, and grows correspondingly in height. (These peaks are significantly blurred at smaller Péclet number, $\mathrm{Pe}=10^{3}$, although we do not show the corresponding results here.) As one can see, the shape of the reconstructed PDF and dissipation rate depend on the number of moments used in the calculations. Although the reconstructed $P(\eta)$ and $W(\eta)$ deviate significantly from corresponding results for the direct numerical simulations, the reconstruction from four even moments resembles the general features of the approximated functions significantly better than the reconstruction from only one (second) moment. The main drawback of the one-moment reconstruction is that it smears out the side peaks and cannot adequately represent poorly mixed regions. The effect of the reconstructed PDF and dissipation rate on the chemical reactions will be discussed below.

The reaction scheme that we use to test our approach is one for which it is well known that the yield can be significantly affected by the details of the mixing process, namely, the two-stage competitive-consecutive reaction ${ }^{17,8}$

$$
A+B \stackrel{k_{1}}{\longrightarrow} R, B+R \stackrel{k_{2}}{\longrightarrow} S,
$$


with initial conditions

$$
(A(0, x, y), B(0, x, y))= \begin{cases}(2,0) & 0 \leqslant x<\frac{1}{2} \\ (0,2) & \frac{1}{2} \leqslant x<1\end{cases}
$$

and $R(0, x, y)=S(0, x, y)=0$ for all $x$ and $y$.

In contrast to the Monte Carlo method, direct solution of the governing equations (2) becomes increasingly computationally expensive as the Péclet number is increased, with well-resolved simulations of (2) being feasible on a desktop PC only up to around $\mathrm{Pe}=10^{5}$. Our method (pseudospectral in space ${ }^{18,19,20}$, with exponential time differencing $^{21}$, specifically the scheme denoted by Cox and Matthews ${ }^{21}$ as ETDRK2) has been described in detail elsewhere ${ }^{8}$, and was chosen for its improved accuracy over other methods $^{19}$. We use either $512 \times 512$ or $768 \times 768$ Fourier modes and a time step of $1.6 \times 10^{-4}$, which both prove adequate for converged results. We have carried out simulations for $\mathrm{Pe}=10^{3}, 10^{4}, 10^{5}$ and for rate constants $k_{1}=10, k_{2}=1$. In this case the reaction is relatively fast in comparison to the mixing time and the outcome of the reaction is sensitive to $\mathrm{Pe}$.

Before comparing results from direct simulation and CMC, we present some scatter plots to demonstrate the close correlation between reactive and passive species, thus to some extent confirming the validity of the underlying assumptions of the CMC model. Fig. 6 shows scatter plots of $R$ against $\eta$, for $\mathrm{Pe}=10^{5}$ and $T=1.6$, after 1 , 2,3 and 4 periods of the flow. If we draw a curve through this scattered points, the deviations of the data points from this curve is smaller than the total variations of the concentration.

Calculations using CMC are performed as follows. We combine Eq. (5) with Eq. (12) to obtain the conservative form of the reaction-advection-diffusion equation in $\eta$-space:

$$
\frac{\partial P Q_{i}}{\partial t}=\frac{\partial}{\partial \eta}\left(-Q_{i} \frac{\partial W}{\partial \eta}+W \frac{\partial Q_{i}}{\partial \eta}\right)+\Omega_{i}(\boldsymbol{Q}) P
$$

which is solved by a finite-difference method as follows. The first and third terms of Eq. (22) are discretized by a Crank-Nicolson scheme similarly to Eq. (17). The second term is approximated by the first-order upwind scheme

$$
Q \frac{\partial W}{\partial \eta} \approx \frac{1}{2}\left(Q_{i}^{j+1}+Q_{i}^{j}\right) \max \left(0, v_{i}^{j+1 / 2}\right)+\frac{1}{2}\left(Q_{i+1}^{j+1}+Q_{i+1}^{j}\right) \min \left(0, v_{i}^{j+1 / 2}\right),
$$

where

$$
v_{i}^{j+1 / 2}=\frac{W_{i+1}^{j+1 / 2}-W_{i}^{j+1 / 2}}{\Delta \eta}
$$

and the upper and lower indices correspond to time and mixture fraction, respectively. The reaction term is modeled by the second-order Adams-Bashforth method. The resulting equations are solved by a tri-diagonal solver. Note that at the beginning of the process $W$ is close to a $\delta$-function and the discretization error in Eqs. (23)-(24) can be relatively high.

The results of our simulations are presented in Fig. 7. To compare the results using CMC methods with the full two-dimensional simulations, we plot spatially averaged 
concentrations of the products, $\langle R\rangle$ and $\langle S\rangle$; then the reactant concentrations $\langle A\rangle$ and $\langle B\rangle$ follow automatically from the material balance. For $T=1.6$ (globally chaotic case), the obtained results have very low sensitivity to the shape of the PDF. Results obtained with only one moment (the second) fit the DNS data as well as results obtained using the first four even moments and so we do not show the former on the figure, for this value of $T$. If the chaotic region coexists with significant regular islands $(T=1.0)$, the shape of the PDF becomes more important. For high Pe, when the chaos becomes the dominant mixing mechanism, the one-moment reconstruction leads to qualitatively wrong results.

To compare our method with the presumed-PDF method, we performed calculations with the $\beta$-PDF method ${ }^{12}$. Due to the symmetry of the initial conditions, the equation for the $\beta$-PDF reads

$$
P(\eta)=\frac{[(1+\eta)(1-\eta)]^{\nu-1}}{\int_{-1}^{1}[(1+s)(1-s)]^{\nu-1} \mathrm{~d} s},
$$

where $\nu=\left(1-M_{2}\right) / 2 M_{2}$. Inspection of Fig. 7 shows that although the presumed-PDF method works reasonably well (but worse than the reconstructed PDF based on only one moment) for the globally chaotic case, the error becomes unacceptably high when $T=1.0$.

In the present work, we model our system as an incompletely stirred reactor (ISR $)^{9}$, i.e., we assume that all conditioned means are the same over the entire flow region. This assumption can be justified in a globally chaotic (or in some classes of turbulent) flow field. Due to the rapid mixing, the PDF approaches a unimodal shape with relatively small standard deviation. In this case the details of the PDF appear to become unimportant - at least, the success of both our calculations and the presumed-PDF method for different combustion models demonstrates low sensitivity of the chemical reactions to the precise shape of PDF. Note that although $P(\eta)$ and $W(\eta)$ can be approximated by a wide range of functions, the dissipation rate has to be consistent with the distribution of $\eta$, i.e., $P(\eta)$ and $W(\eta)$ must satisfy Eq. (12). If the chaotic region coexists with poorly mixed islands, the assumptions which lead to the ISR model are no longer valid, and the approximate modelling of the PDF gives incorrect predictions of the outcome of the reaction. Thus it is interesting to note that a more detailed representation of the PDF captures the neglected spatial variations of the concentrations. One of the obstacles for numerical implementation of the CMC method is the additional dimensionality associated with the conditioning variable. It has been noted $^{9}$ that the CMC method can use a much coarser spatial grid than that in a CFD solver. Our results show that the spatial resolution and the resolution in the mixture fraction space mutually complement one another, and that significant inhomogeneity in physical space can be successfully captured by corresponding variations along the $\eta$ axis. 


\section{Conclusions}

A new approach to the simulation of chemical reactions in laminar chaotic flow has been proposed, in which a Monte Carlo method provides the statistical moments of an associated passive scalar and the CMC method is used to model the chemical reaction. Comparison with direct numerical simulation shows that the approach provides an accurate description of the chemical reactions when the underlying flow field has no significant nonchaotic regions.

The salient component of the proposed methodology linking the Monte Carlo and $\mathrm{CMC}$ methods is the reconstruction procedure, which produces the PDF and dissipation rate of the passive scalar with given statistical properties. Although in the present study we use the results of statistical simulations as input to the reconstruction procedure, Monte Carlo is not the only available method which can provide the necessary statistical information. Any other data obtained by experimental and/or numerical methods can be incorporated into the proposed scheme, which then can be applied to turbulent as well as to laminar flows.

In the present study, we have treated the flow region as a single-chamber reactor. Note that the incompletely stirred reactor model ${ }^{9}$ has been proposed for a region of the flow bounded by walls across which fluxes are known. However, if we consider a series of reactors where the outlet of $i$ th reactor serves as inlet of $j$ th reactor the proposed method can readily be extended to a compartment model. We denote the conditional flux from $i$ th to $j$ th compartment by $U_{i j}(\eta)$, where $U_{i j} \geqslant 0$; then the total mass flux between these two compartments is

$$
\int\left(U_{i j}(\eta)-U_{j i}(\eta)\right) \mathrm{d} \eta
$$

Further, we denote by $V_{i}, P_{i}(\eta)$ and $N_{i}^{2}(\eta)$ the volume of the $i$ th compartment, the PDF of the passive scalar in the $i$ th compartment and its conditional dissipation rate, respectively. The multi-compartment analog of Eq. (7) then reads

$$
V_{i} \frac{\partial P_{i}(\eta)}{\partial t}+\sum_{j} P_{i}(\eta) U_{i j}(\eta)-\sum_{j} U_{j i}(\eta) P_{j}(\eta)=-V_{i} \frac{\partial^{2} N_{i}^{2}(\eta) P_{i}(\eta)}{\partial \eta^{2}} .
$$

Therefore, as in the one-compartment case, we have to find a set of nonnegative functions $P_{i}(\eta), \mathcal{U}_{i j}(\eta)=P_{i}(\eta) U_{i j}(\eta)$ and $W_{i}(\eta)=N_{i}^{2}(\eta) P_{i}(\eta)$ which minimizes an appropriately defined residual. Since Eq. (27) is linear with respect to $P_{i}(\eta), \mathcal{U}_{i j}(\eta)$ and $W_{i}(\eta)$, the problem can be solved by a standard method for quadratic programming.

\section{References}

[1] Stone HA, Strook AD, Ajdari A. Engineering flows in small devices: Microfluidics toward a lab-on-a-chip. Annu Rev Fluid Mech. 2004;36:381-411.

[2] Aref H. Stirring by chaotic advection. J Fluid Mech. 1984;143:1-21. 
[3] Ottino JM. The kinematics of mixing: stretching, chaos and transport. Cambridge, UK: Cambridge University Press; 1989.

[4] Aref H. The development of chaotic advection. Phys Fluids. 2002;14:1315-1325.

[5] Muzzio FJ, Ottino JM. Dynamics of a lamellar system with diffusion and reaction: Scaling analysis and global kinetics. Phys Rev A. 1989;40:7182-7192.

[6] Muzzio FJ, Ottino JM. Diffusion and reaction in a lamellar system: Self-similarity with finite rates of reaction. Phys Rev A. 1990;42:5873-5884.

[7] Ottino JM. Mixing and chemical reactions: a tutorial. Chem Eng Sci. 1994;49:4005-4027.

[8] Cox SM. Chaotic mixing of a competitive-consecutive reaction. Physica D. 2004;199:369-386.

[9] Klimenko AY, Bilger RW. Conditional moment closure for turbulent combustion. Prog Energy Combust Sci. 1999;25:595-687.

[10] Devaud CB, Bilger RW, Liu T. A new method of modeling the conditional scalar dissipation rate. Phys Fluids. 2004;16:2004-2011.

[11] Mortensen M. Consistent modeling of scalar mixing for presumed, multiple parameter probability density functions. Phys Fluids. 2005;17:018106.

[12] Mortensen M. Implementation of a conditional moment closure for mixing sensitive reactions. Chem Eng Sci. 2004;59:5709-5723.

[13] Vikhansky A. Quantification of reactive mixing in laminar microflows. Phys Fluids. 2004;12:4738-4741.

[14] Vikhansky A, Cox SM. Reduced models of chemical reaction in chaotic flows. Phys Fluids. 2006;18:037102.

[15] Vikhansky A. Coarse-grained simulation of chaotic mixing in laminar flows. Phys Rev E. 2006;73:056707.

[16] Franjione JG, Ottino JM. Symmetry concepts for the geometric analysis of mixing flows. Phil Trans R Soc London, Ser A. 1992;338:301-323.

[17] Levenspiel O. Chemical reaction engineering. New York, USA: Wiley; 1972.

[18] Giona M, Cerbelli S, Adrover A. Quantitative analysis of mixing structures in chaotic flows generated by infinitely fast reactions in the presence of diffusion. $J$ Phys Chem. 2002;106:5722-5736.

[19] Adrover A, Cerbelli S, Giona M. A spectral approach to reaction/diffusion kinetics in chaotic flows. Comp Chem Eng. 2002;26:125-139. 
[20] Giona M, Cerbelli S, Adrover A. Geometry of reaction interfaces in chaotic flows. Phys Rev Lett. 2002;88:024501.

[21] Cox SM, Matthews PC. Exponential time differencing for stiff systems. J Comp Phys. 2002;176:430-455. 


\section{List of figure captions}

Fig 1. Solution of a test problem: reconstruction, based on second and fourth moments, of (a) the fractional dissipation rate $W$ and (b) the PDF $P$. The finite-difference method smears the $\delta$-function over several neighboring cells.

Fig 2. Sine flow (18): flow field during first (left) and second (right) half-period.

Fig 3. Grayscale plots of $Z$, evolving in the sine flow (18) with $T=1.0$. Results are obtained from direct simulation of (3), from the initial condition (19). Black and white correspond, respectively, to $Z=+1$ and $Z=-1$. The upper sequence corresponds to $\mathrm{Pe}=10^{5}$ and the lower sequence to $\mathrm{Pe}=10^{3}$. Plots show $Z$ at times $n T / 2$ for $n=1, \ldots, 12$.

Fig 4. From top to bottom: second, fourth, sixth and eighth moments of the passive scalar $Z$. Symbols correspond to the Monte Carlo results, while lines are from the interpolation procedure. $T=1.6, \mathrm{Pe}=10^{5}$.

Fig 5. PDF (left column) and dissipation rate (right column) after 2, 4, 6, 8 and 10 periods (from top to bottom). Shown are results from direct numerical simulation (solid lines), reconstruction with first four even moments (dashed lines) and reconstruction with only one (second) moment (dotted lines). $\mathrm{Pe}=10^{5}, T=1.0$.

Fig 6. Scatter plots of $R$ against $\eta$ for $\mathrm{Pe}=10^{5}$ and $T=1.6$ after (a) 1, (b) 2, (c) 3, (d) 4 periods of the flow.

Fig 7. Results for $k_{1}=10, k_{2}=1$ : direct numerical simulation (solid line); CMC with PDF reconstructed with four even moments (diamonds); CMC with PDF reconstructed with only the second moment (hexagons); presumed $\beta$-PDF (circles). Concentrations of $R$ (upper lines) and $S$ (lower lines) for $\mathrm{Pe}=10^{3}, 10^{4}, 10^{5}$ (from top to bottom). $T=1.6$ (left column) and $T=1.0$ (right column). 
(a)

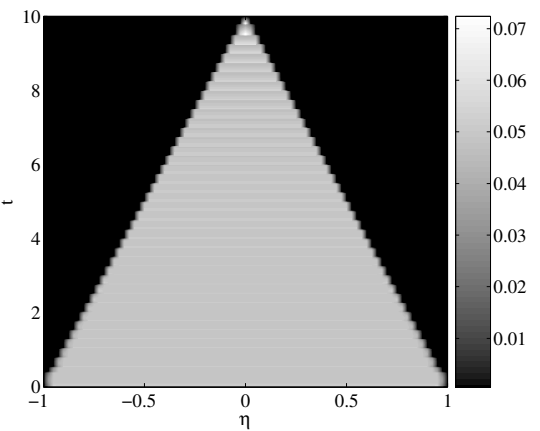

(b)

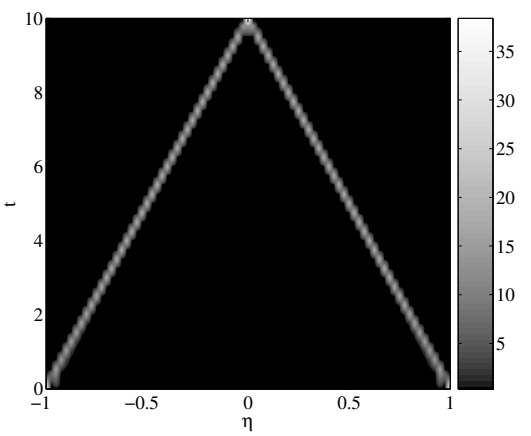

Figure 1: Solution of a test problem: reconstruction, based on second and fourth moments, of (a) the fractional dissipation rate $W$ and (b) the PDF $P$. The finite-difference method smears the $\delta$-function over several neighboring cells. 

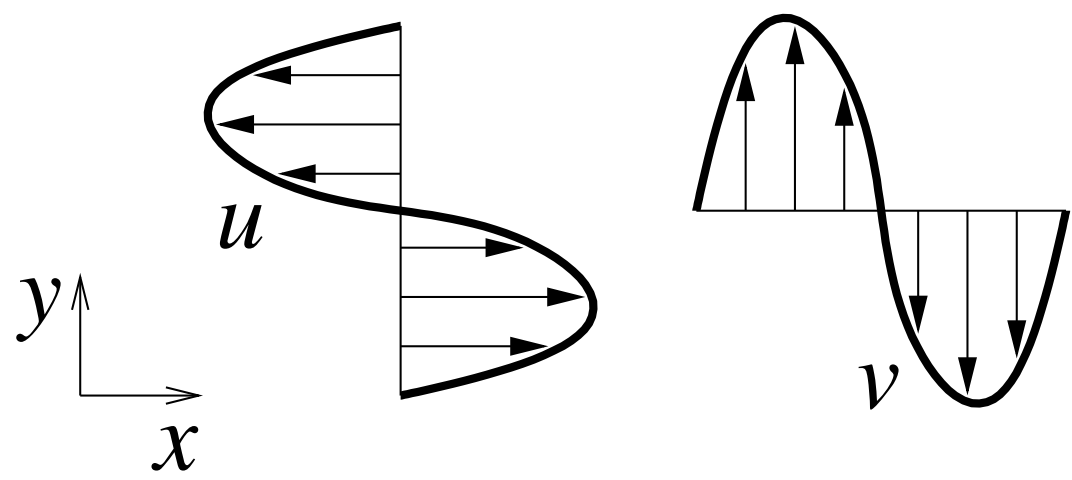

Figure 2: Sine flow (18): flow field during first (left) and second (right) half-period. 

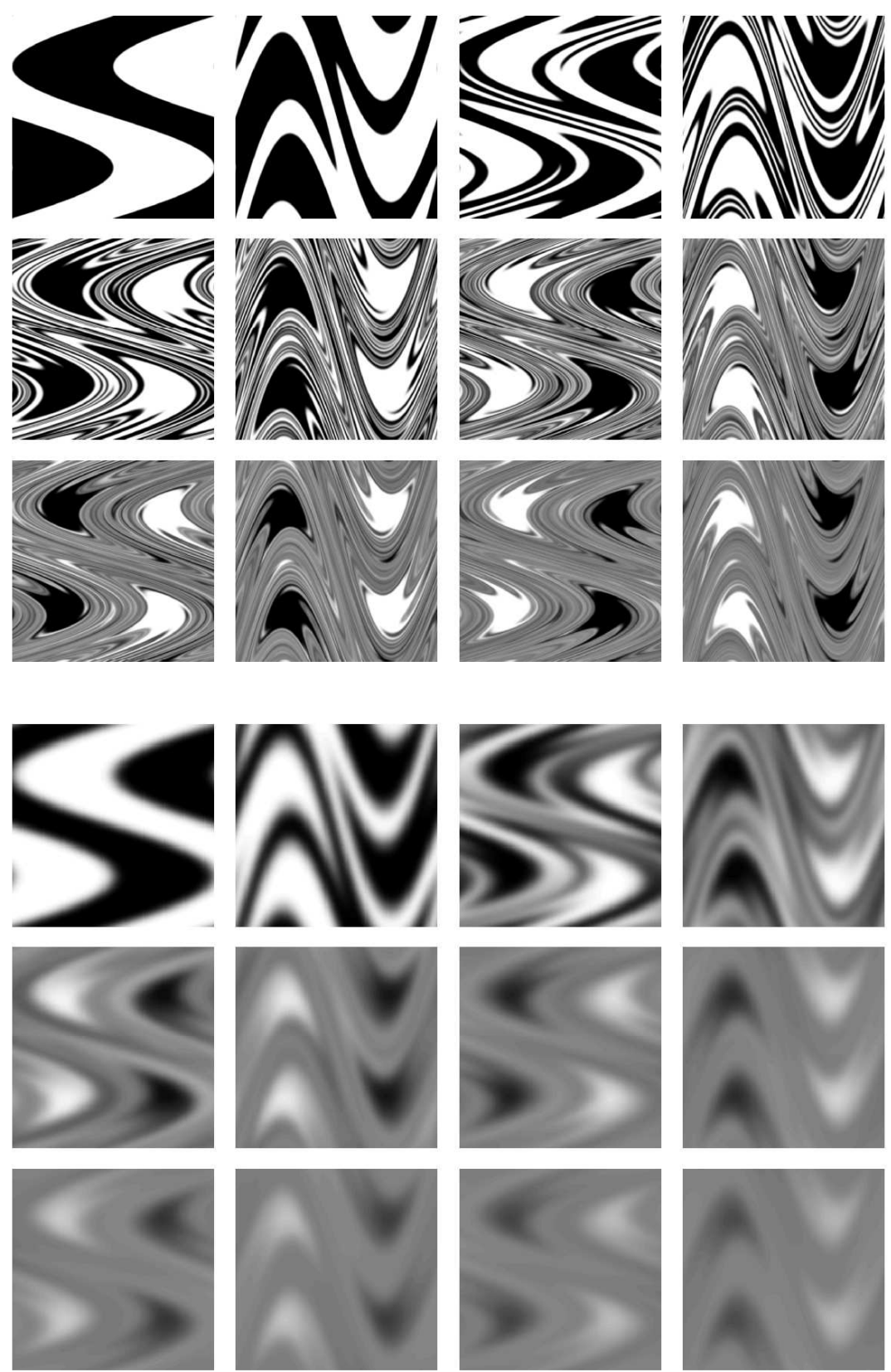

Figure 3: Grayscale plots of $Z$, evolving in the sine flow (18) with $T=1.0$. Results are obtained from direct simulation of (3), from the initial condition (19). Black and white correspond, respectively, to $Z=+1$ and $Z=-1$. The upper sequence corresponds to $\mathrm{Pe}=10^{5}$ and the lower sequence to $\mathrm{Pe}=$ $10^{3}$. Plots show $Z$ at times $n T / 2$ for $n=1, \ldots, 12$. 


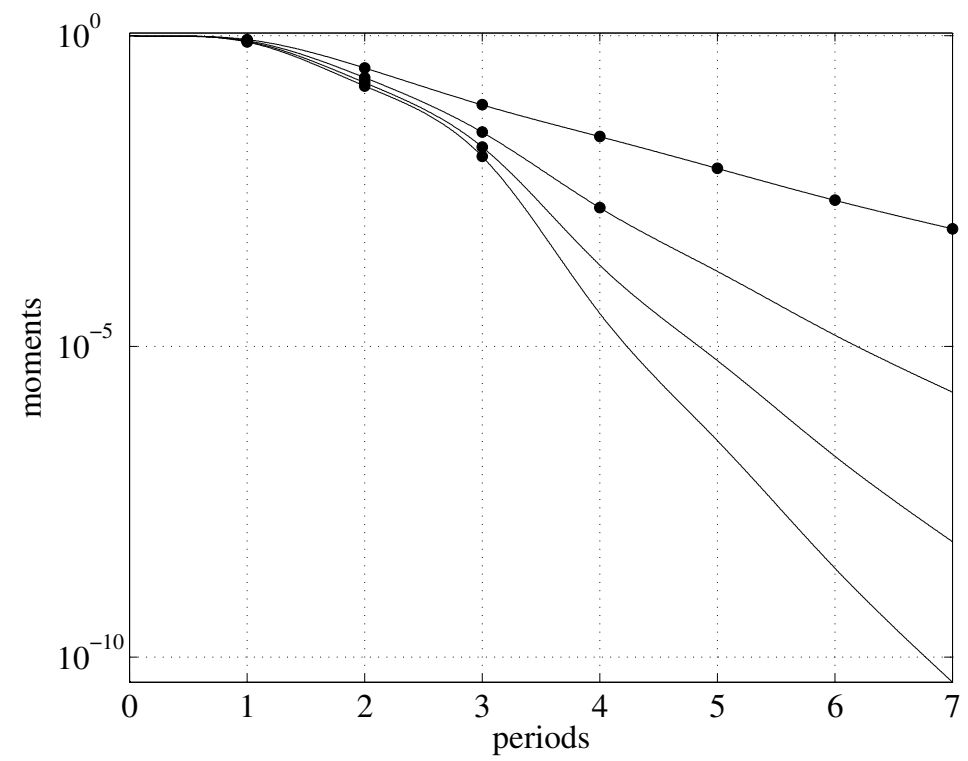

Figure 4: From top to bottom: second, fourth, sixth and eighth moments of the passive scalar $Z$. Symbols correspond to the Monte Carlo results, while lines are from the interpolation procedure. $T=1.6, \mathrm{Pe}=10^{5}$. 
(a)

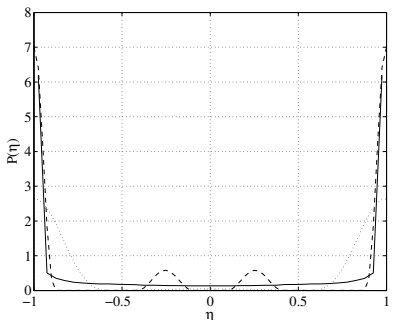

(b)

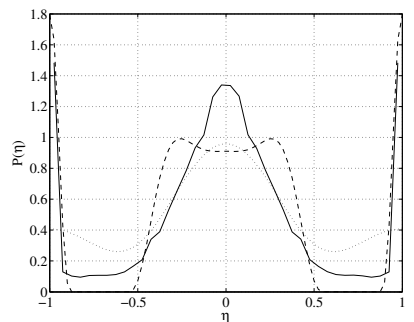

(c)

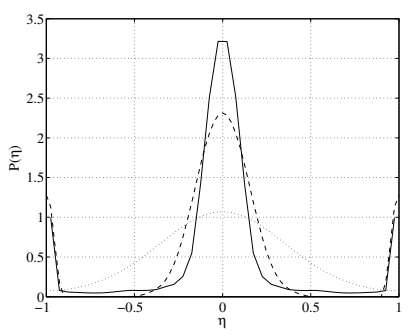

(d)

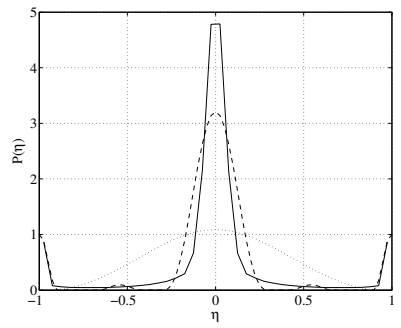

(e)

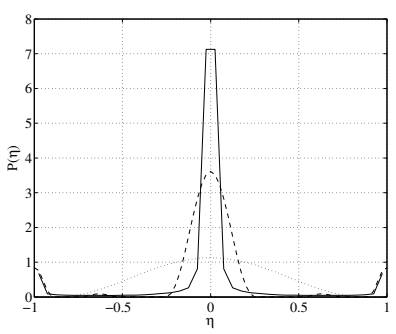

(f)

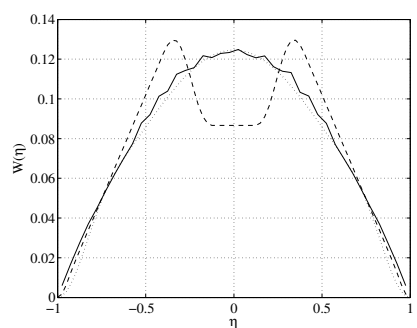

(g)

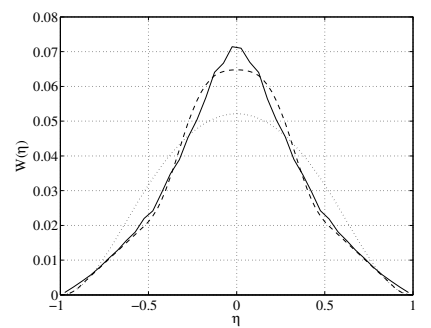

(h)

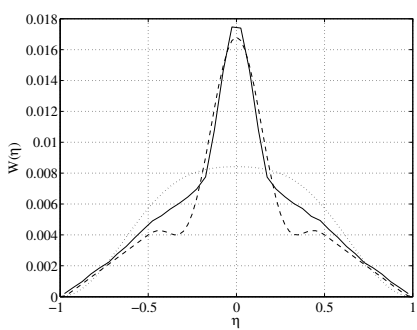

(i)

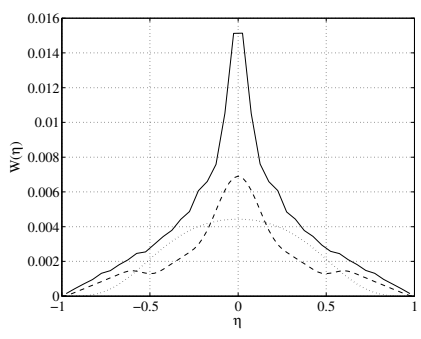

(j)

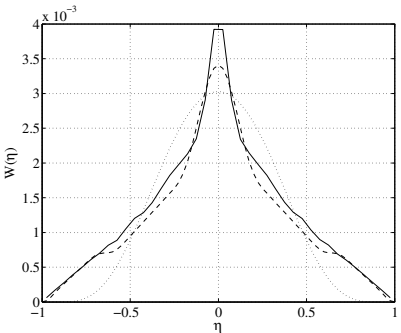

Figure 5: PDF (left column) and dissipation rate (right column) after 2, 4, 6, 8 and 10 periods (from top to bottom). Shown are results from direct numerical simulation (solid lines), reconstruction with first four even moments (dashed lines) and reconstruction with only one (second) moment (dotted lines). $\mathrm{Pe}=10^{5}, T=1.0$. 
(a)

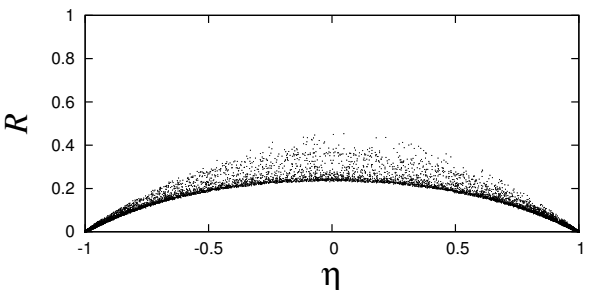

(b)

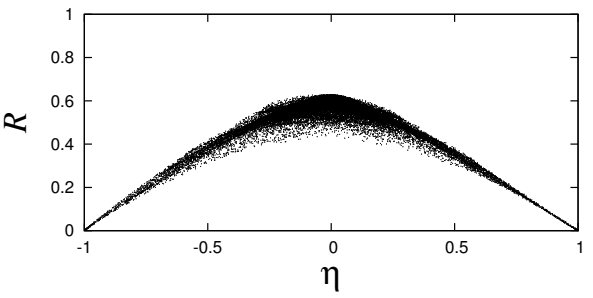

(c)

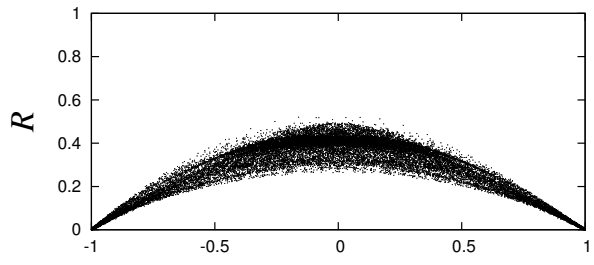

(d)

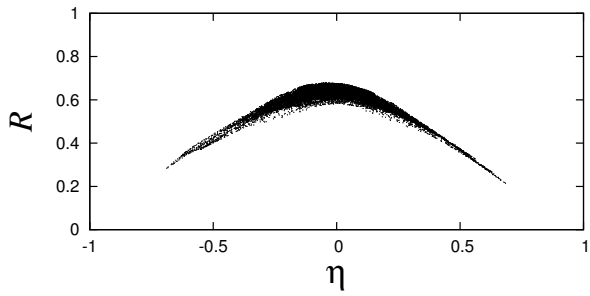

Figure 6: Scatter plots of $R$ against $\eta$ for $\mathrm{Pe}=10^{5}$ and $T=1.6$ after (a) 1, (b) 2, (c) 3, (d) 4 periods of the flow. 
(a)

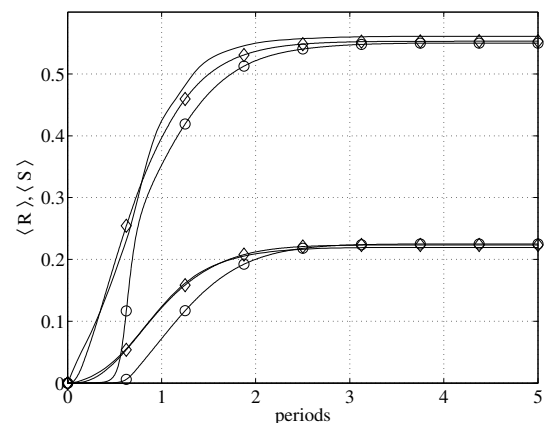

(b)

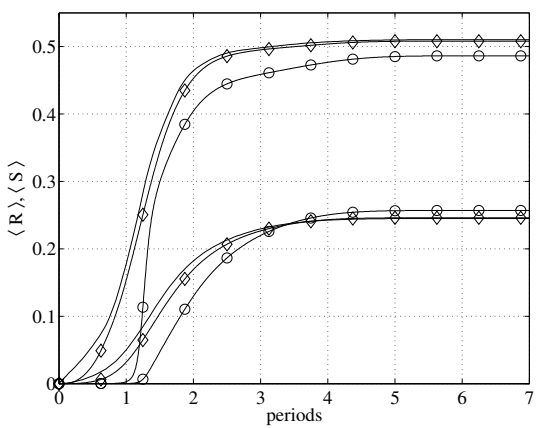

(c)

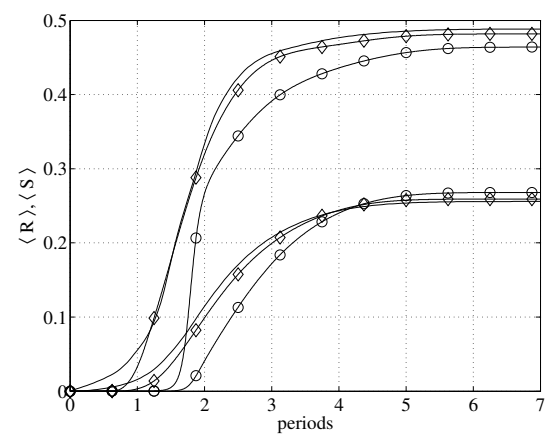

(d)

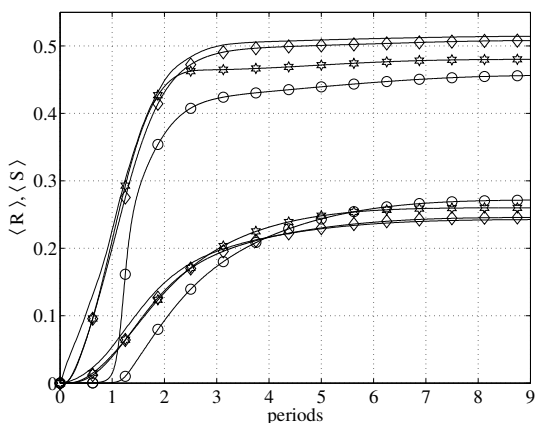

(e)
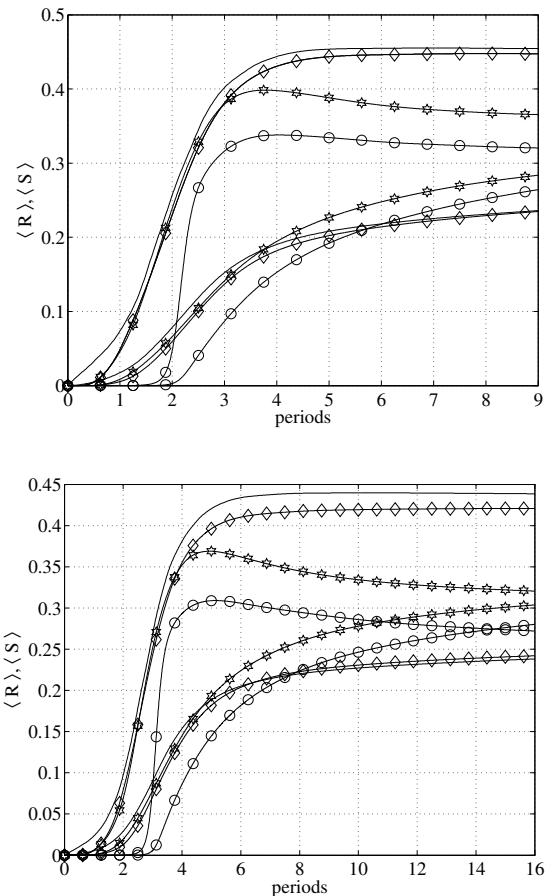

Figure 7: Results for $k_{1}=10, k_{2}=1$ : direct numerical simulation (solid line); CMC with PDF reconstructed with four even moments (diamonds); CMC with PDF reconstructed with only the second moment (hexagons); presumed $\beta$-PDF (circles). Concentrations of $R$ (upper lines) and $S$ (lower lines) for $\mathrm{Pe}=10^{3}, 10^{4}, 10^{5}$ (from top to bottom). $T=1.6$ (left column) and $T=1.0$ (right column). 